\title{
The technology of variable message format simulation in software testing
}

\author{
Fei.Gao ${ }^{1, a}$, Xuejun. $\mathrm{Bi}^{1, \mathrm{a}}$ and Xiaozhen. Wang ${ }^{1, \mathrm{a}}$ \\ Academy of Armored Force Engineering, Beijing 100072, China
}

Keywords: Interface testing, Short message transmission, Variable message format simulation

\begin{abstract}
The complexity of software upgraded is the inevitable result of the deepening of social information, and then to put forward higher requirements of software testing. Because of technology, co-ordination, geopolitical, or other non-technical factors these factors the tested software and related hardware devices can not connect to the system, leading to delay the progress of the test, the level of systemic problems in the design of feedback difficulties. This paper presents a short message format with variable packet transmission simulation methods required for devices connected to the radio transmission of images, for example, through variable message format technology to the system under test and simulation data transmission between stations, measured test between serial communication port terminal and radio interfacing and get results.
\end{abstract}

\section{THE Research background}

\subsection{The testing problem with the hardware}

Software testing is an important part of the software life cycle indispensable, as the software complexity continues to increase, many systematic software have to with the appropriate hardware to play its due effect. In actual testing process, due to co-ordinate scheduling, third-party testing platform and hardware vendors to provide location gap is too far away, a particular device under control and other factors in a particular situation, leading to delay the progress of the test, and to influence the test of the robustness The author tests had witnessed several cases above on several software tests. While connected to the corresponding hardware test irreplaceable data transmission can not be replaced, through simulation tests can be found on the problems of software design has been good and bad feedback.

\subsection{Traditional test system structure}

In this paper, a software testing project which author participate in will be shown as an instance. For example, the system structure shown in Figure 1. The measured software via serial communication port is connected with the radio station, the radio station transmission by wireless radio station to communicate with another, the same radio station connected via serial communication port and test the software on another set of equipment.

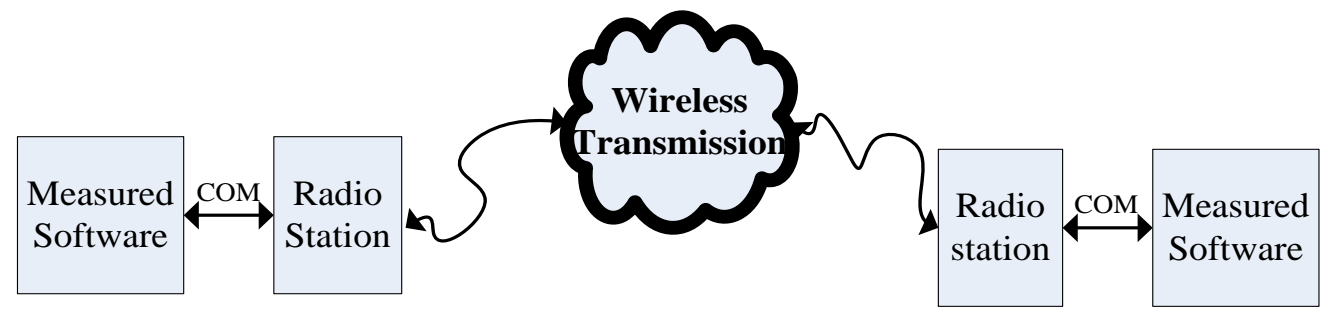

Fig. 1 The traditional test system structure

\subsection{The paper structure}

The first section describes the background; The second section proposes a new system design ideas; The third section describes the advantages of using variable message format simulation; The forth section raises how to build test environment up; The fifth section states simulation test results are given; The sixth second is summarizes. 


\section{New test system structure}

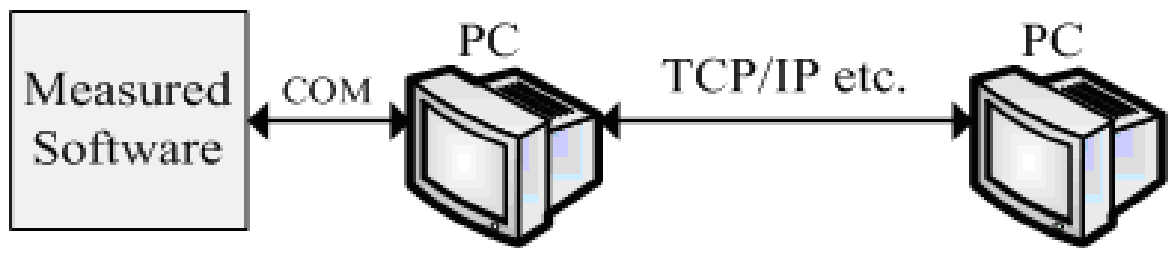

Fig. 2 The new system structure

As shown in figure 2, the PC will be used to replace the original radio station, with the TCP / IP network in place of wireless transmission. This structure can be greatly improved over conventional test configuration, the dependence of the hardware is greatly reduced than the original system. The original system structure in the testing process will take some manpower and resources to ensure the normal operation of the hardware, the new architecture can optimize staffing and better testing. The picture encapsulates the target packet header additional applications, both in the corresponding process is complete standards. When sent via radio should follow the SLIP (Serial Line Internet Protocol).

\section{Using VMF Transmission}

\subsection{VMF virtues}

The four advantages are listed below:

(1)Information coding standard of variable message format is bit-oriented. Messages are passed vie binary code. It can not only reduce the amount of data transmitted, but also improve machine automatically recognize and deal with the message easily.

(2)Because of its coding standards, VMF can make use of network resource to enhance the adaptability and effectiveness of the message.

(3)According to the literal meaning of VMF, the specific format and length of VMF can be adjusted in practical application.

(4)Because of its flexible syntax, VMF only allow users to send necessary information. The size of the necessary information could be changed, thereby reducing the amount of data transmitted and saving bandwidths.

\subsection{An example of VMF}

The following table gives a practical example VMF message structure can be seen in the table, a liberal news telegrams from a FRI indicator and a repeat of the text field structure, the message can be transmitted up to 6 length more than 2048 characters string information. Such as through a natural language telegraph message transmission content " request air support" the text information, the text information is encoded as "8E D7 8F E7 77 E1 83 4D AD 4F A9 5D $0100 " 14$ bytes of binary data, visible, as opposed to a fixed length packet format, VMF message formats can be automatically adjusted according to the transmission of data encoded content length, the maximum savings transmission channel resources.

Table 1 Free Text Message

\begin{tabular}{llllll}
\hline Index & Number & DFI/DUI & DUI Name & Bits & Type \\
\hline 1.1 & & 4045002 & FRI & 1 & $\mathrm{M}$ \\
1.2 & & 4265001 & Free Text & $2048 \mathrm{M}$ \\
Index & Number & Group Code & Repeat Code & Explain \\
1.1 & & & R1 (6) & Repeat6 times \\
1.2 & & & R1 & \\
\hline
\end{tabular}




\section{Variable message format simulation test process}

Logical flow test is shown below, with radio transmission picture as an example: the measured terminal picture packets encapsulated into the goal, the programming will be added in front of the goal on the attached application packet header. Target messages and additional application packet should follow certain message standards, this does not explain in detail unfold. The message to the short message server, which follows the no connection reliable transport protocol. Following the adoption of the radio transmission station transmits follow SLIP protocol. Issued via serial communication port.

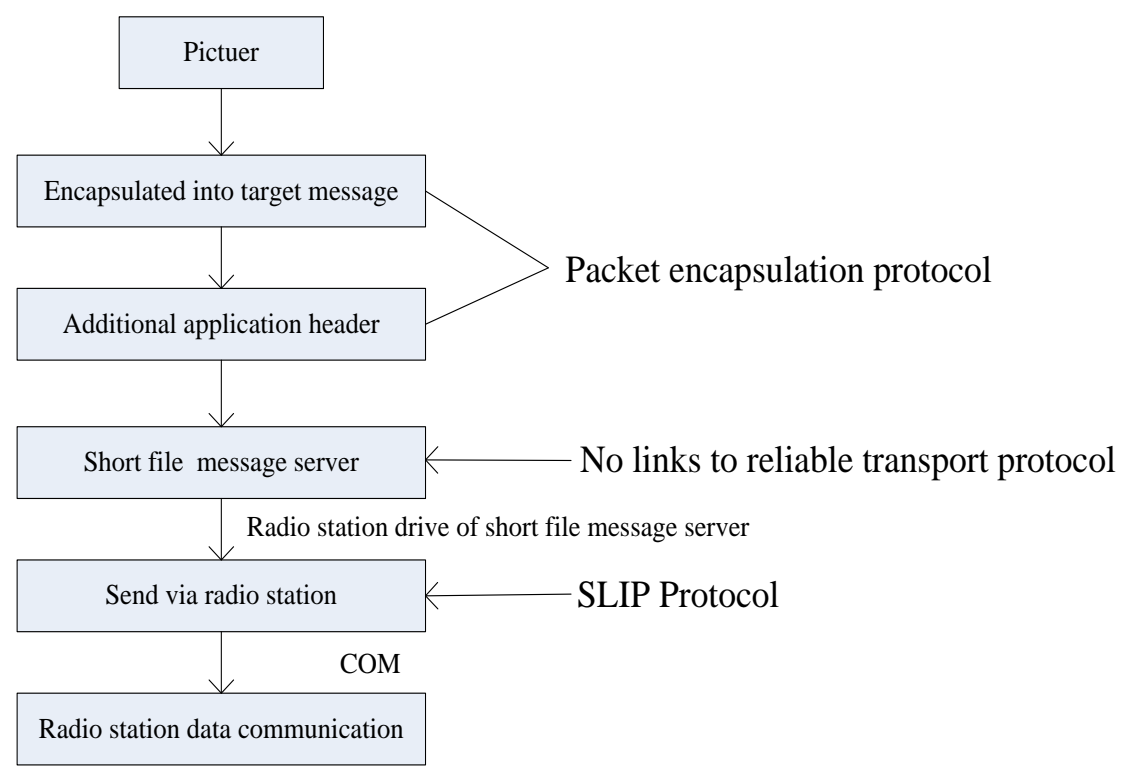

Fig. 3 The process of simulation test

\subsection{Construction of protocol agreement}

As shown in the figure 4, the application layer has no links to reliable transport protocol consists of head, application layer head and user data. Each data block has terminal segment number. Data at the receiving end according to the late period of number to estimate the time required to receive the all data block. According to the estimate of the time, data at the receiving end set the timeout timer. If before the timer timeout to receive the all data segment, the receiving end will complete a segmented restructuring to the upper application, and sends the complete confirmation to the sender; otherwise, the receiving end sends sectional confirmation to confirm the segment, which is received correctly, the sending end will send those segment again.

\begin{tabular}{|c|c|c|}
\multicolumn{2}{c}{ Low byte } & High byte \\
\hline Head & Application layer head & User data \\
\hline
\end{tabular}

Fig. 4 Structure of data unit

\subsection{Repeatable processing of data items}

In the variable message format messages, data items can use FRI or GRI indicator control. If the indicator value is 0 , the data item indicated is the last time; otherwise, it is meaning that after the item has the same data content. As a result, the program use the following structure template to represent data items can be repeated. 


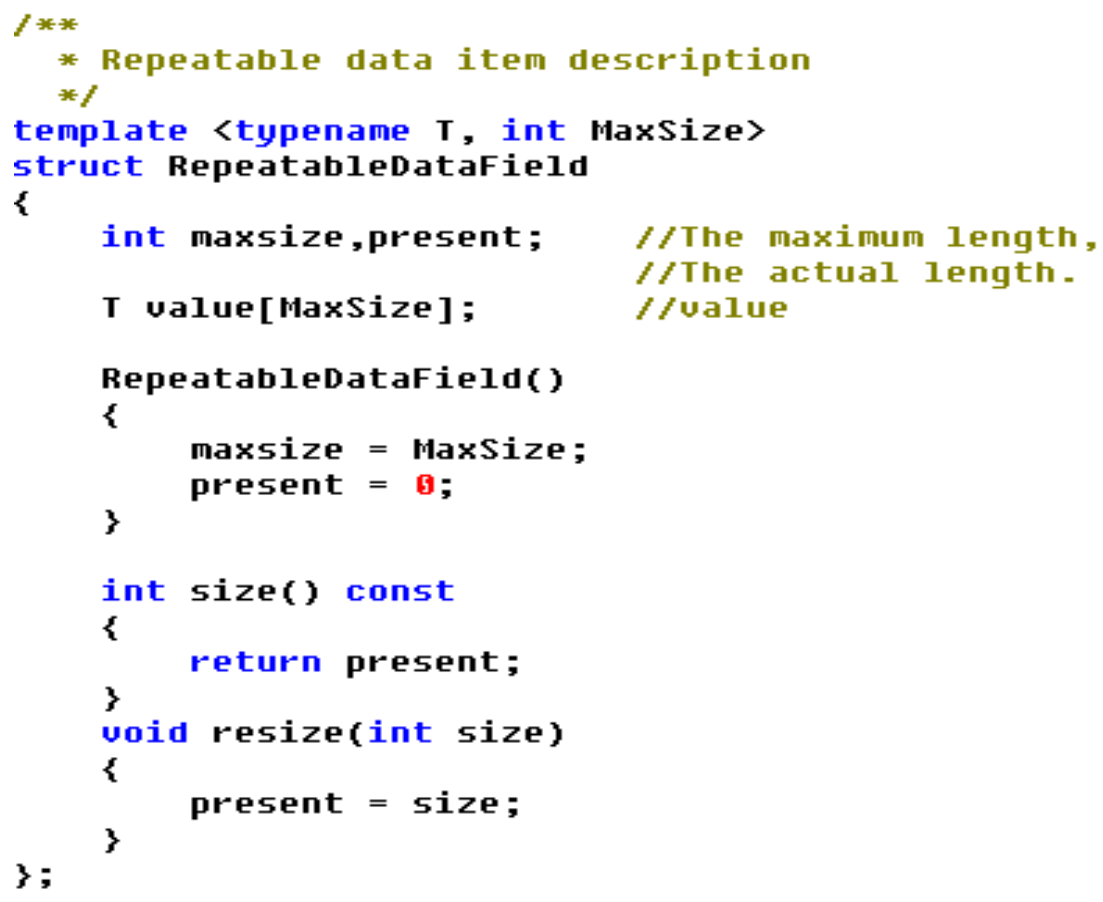

Fig. 5 Repeatable data item description template

\section{Example of radio transmission image}

Practical examples of radio transmission pictures

(1): will a serial adapter cable (serial cable to the monitor terminal sequence with the computer program to convert a serial line) access test terminal end "serial", the other end to the other serial cable, the other port the other end of the cable connected to the computer serial port;

(2): Run PC serial debugging assistant (serial master, ComMaster1.1 for Win2K / XP) set the baud rate 115200bit, 8 data bits, parity bit is NO, 1 stop bit; in the "Advanced Options" setting: Hardware RTS / CTS flow control;

(3): operation monitoring terminal software, enter the "wireless transmission" Send a picture: filename test.jp2, file size is $7 \mathrm{~K}$.

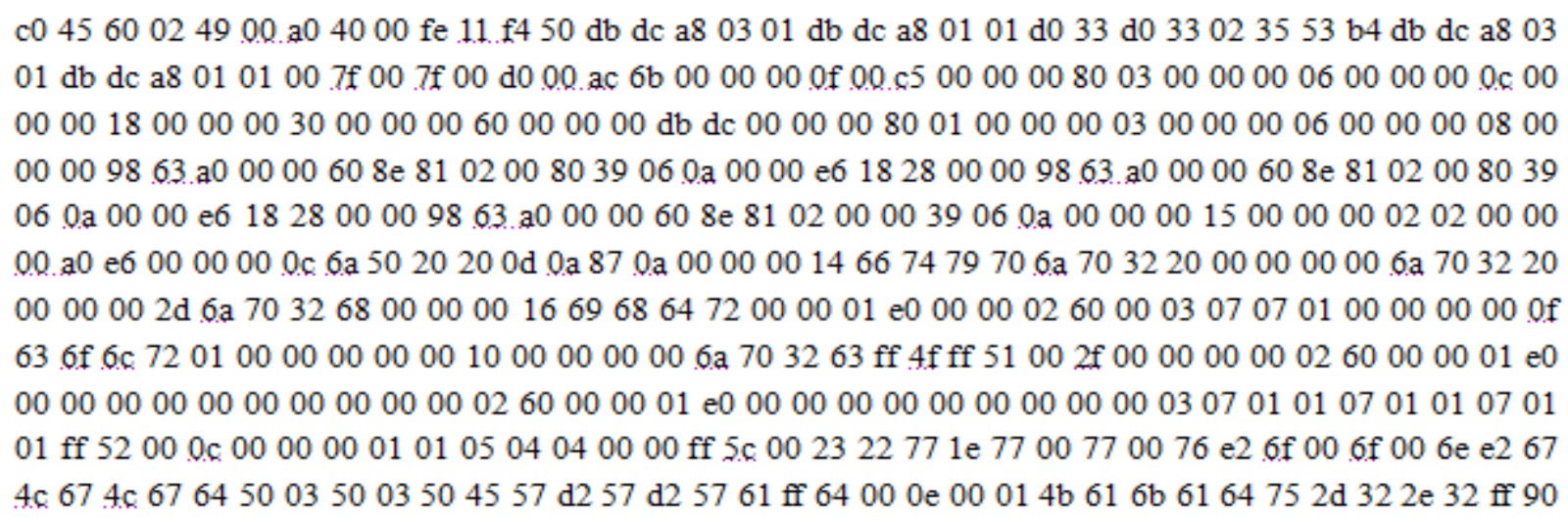

Fig. 6 The terminal receives the binary message(part)

Problem Description: The serial assistant, capture data terminal in the process of sending tactical picture given to the radio station, according to the design specifications, reference SLIP protocol and short message transfer protocol parsing out the picture packet header and its applications from the captured data, data is written to the packet1.dejcb file by VMF packet analysis tool for data analysis, found the application header portion of the following questions:

(1):The destination address repeated 11 times, and the contents are the same

(2):The content of the message processing group repeated 11 times, and the contents are the same as the above phenomena do not meet the messaging during the normal rules. 


\section{Summary}

In this paper, variable message format technology to simulate the measured data transmission between software and related hardware, to achieve the following innovations:

(1): when hardware temporarily unable to connect on a certain systematic testing, though not a substitute for systematic testing, but also on the top-level design has some feedback significance;

(2): The simulation makes the testing process more simple and feasible, to avoid the impact of short-term factors irreconcilable test schedule.

I believe that this method has a good prospect, but research on the Standardization of packet protocols, as well as continue to be thorough, the only way to play the commercial value of its due.

\section{References}

[1]. Wang Chonggang,Kazem Sohraby,Hu Yueming,et.2005. a1.Issues of transport control protocols for wireless sensor networks[C].Proceedings of International Conference on Communications, Circuits and System(ICCCAS),5(1):422-426.

[2]. Chandran K,Raghunathan S,Venkatesan S,et.2001. a1.A feedback-based scheme for improving TCP performance in ad hoc wireless networks[J].IEEE Personal Communications Magazine,8(1):34-39.

[3]. Liu Wenyong, Qi Feng, Chen Yinghui.2010.Graphical Presentation Format of Transaction Model for General Network Management Interface Test, Nanjing, ICCT.

[4]. IEEE Std 829-1998.IEEE standard for software test documentation[S].

[5]. Li Z,Tian J.2003.Testing the suitability of Markov chains as web usage models[C].Proceedings of the 27th Annual International Computer Software and Applications Conference.

[6]. Dusseau D, Brock C.2004.Network centric interoperability-using a variable message based data link to improve situation awareness and close air support[J].IEEE A \& E Magazine,19(9):8-13.

[7]. C20301-6000, Joint Tactical Data Link Management Plan[S]. 